\title{
A Comparative Study on PMIPv6 and Partially DMM Network Architecture
}

\author{
Ye Wang ${ }^{1}$, Xiaobo $\mathrm{Li}^{2, *}$ and Wenhui Zhang ${ }^{3}$ \\ 1Dept. of Electronic and Information Engineering, Lishui University, China \\ 2Dept. of Computer Science and Technology, Lishui University, China \\ 3Dept. of Mechanical Engineering, Lishui University, China \\ 1wychina_2007@hotmail.com,2lxb@lsu.edu.cn,3hit_zwh@126.com,
}

\begin{abstract}
This paper provides A performance analysis and implementation between Proxy Mobile IPv6 (PMIPv6) and partially distributed mobility management (DMM) network architecture. The simulation results indicated DMM networks perform better benefits than PMIPv6 network by analyzing packet delivery ratio (PDR) and CPU point-to-point utilization.

Keywords: PMIPv6, DMM, PDR, utilization

\section{Introduction}

With the increasing volumes of mobile data traffic and massive increase in the number of interconnected devices, espectally on demand,for "imperceptible latency" with tactile internet, and nearly 100\% reliability with Internet of Thing service [1], IMT-2020 (5G) provides a Fifth-Generatio (5G) systemnto neet new and unprecedented demands. Along with these objectives, distributed mobility management (DMM) has recently emerged as a new paradigm to desigh a flat and flexible mobility architecture, allowing traffic to be broken out locally closer to the edge (i.e., offloading the network core) and exploiting the use of different gateways for traffic with different connectivity and mobility requirements. Apparently, the DMM approach is a suitable candidate for mobility management in future $5 \mathrm{G}$ dense deployments [2]

A number of distibuted mobility management schemes have been proposed in literature. For the requirements and DMM network architecture issue, Liu et al. [35] defined the requirements and provided a flattened 3GPP network mechanism, while Chan et al. [6] introduced several SIPTO/LIPA scenarios based on DMM. For the Mobile Node's (MN) location management issue, Giust et al. [2,7] and Bernardos et al. [8] proposed a method by querying the Central Mobility Database (CMD) and Software Define Network (SDN) and Domain Name System (DNS) server to acquire the MN's location information. Kim et al. [9] and Jung et al. [10] proposed a multicast or point-to-point search mechanism to find an MN's location. For the MN's data flow issue, Sun et al. [11] presented a novel solution that supported different IP data flow by multiple IP interface. However, those proposed mechanisms did not give a specific implementation mechanism and feasibility analysis. For the performance analysis issue, Shin et al. [12] analyzed the simulation data which showed DMM mechanism is more suitable for sensitive delay and tolerance than Proxy MIPv6. Giust et al. [13] and Kim et al. [14-15] showed signal control and data transmission cost analysis. However, those performances are all cost-based analyses, which are short of the comprehensive and systematic study. ${ }^{1}$
\end{abstract}

11 *Corresponding Author: Xiaobo Li, Email: 1xb@1su.edu.cn 
In this paper, the partially distributed mobility management (DMM) network and PMIPv6 network were evaluated in OPNET simulator [16] by analyzing the different performance metrics, such as packet delivery ratio (PDR) and CPU pointto-point utilization. The simulation results indicated that performance analysis of DMM network performs better than PMIPv6 network.

\section{Network Architecture and Simulation Methods}

\subsection{Overview of Partially Distributed Mobility Management}

DMM aims to adapt existing IP mobility protocols such as MIPv6 and PMIPv6 to the emerging flat IPv6 mobile networks architectures. It intends to distribute and confine the data plane and control plane at the Access Routers (ARs) level The partially DMM mechanism only separates the data plane, where the DMIARs will manage the data without going through the CMD. In addition, DMM is expected to reduce the tunneling overhead, global network signaling loads, packet transmission cost and end-to-end delay, etc.

As PMIPv6-based partially DMM approach, shown in Figure 1, which is required to implement the DMM requirements at each ARs. Hence, it is guaranteed that the MN is always attached to an AR that can act as a mobility anchor, named Distributed Mobility Anchor Router (DMAR). This allows the MN to always initiate new sessions using the current IP address'[17]. The lata traffic is then routed optimally without tunneling. While MN undergoes an IP handover before terminating these data sessions, then DMARS w11 exchange the distributed proxy binding update (d-PBA) and distributed proxy binding acknowledgement (d-PBA) message with the CMD, a tunnel is then established between the new DMAR2 and previous DMAR1. In order to be able to send a d-PBU from new DMAR2 and CMD, the binding cache and biding apdate list should be created in corresponding DMARs and CMD. ${ }^{\circ}$

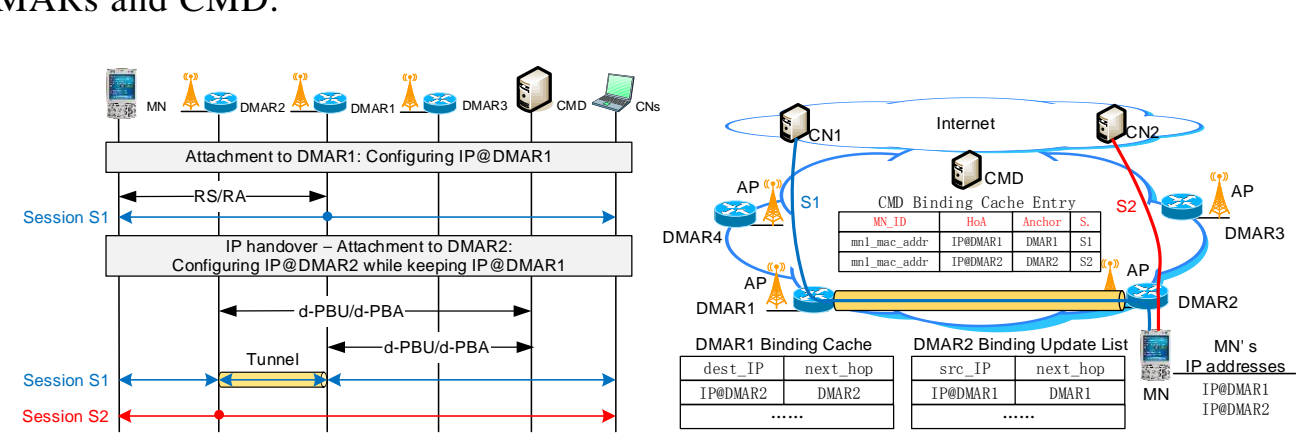

Figure 1. Mobility Management in Pmipv6-Based Partially DMM 


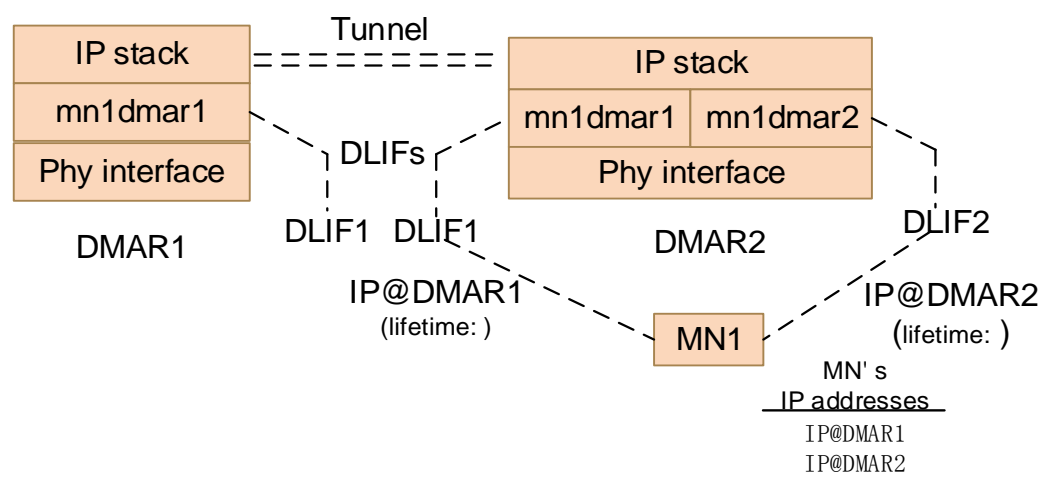

Figure 2. A Proposed MN's Detection and Data Forward Moojel

One of the main challenges of network-based DMM solution is how a DMAR knows MN's attachment or handover and how to allow an MN to simultaneously send/receive traffic which is anchored at differennARs? This paper proposed an MN's detection and data forward policy model, as shown in Figure 2. The distributed logical interface (DLIF) concept [18] is modeled in the proposed scheme. Whenever MN undergoes an IP handover or attachment in DMARs, DMARs create a distributed logical interface to communicate (point-point link) with $\mathrm{MN}$, exposing itself as a logical router with a specific MAC address (e.g., 36:02:86:23:08:01) and IPv6 addresses(1P@DMARs) using DLIF (mn1dmars). The IP sessions will be maintained based on the session lifetime.

\subsection{Overview of Difference between Pmipwg and DMM}

The main difference between PMIP16 and partially DMM network, shown in Figure 3, is the partially distributed category, which consists of de-coupling the entities that participate in the data and controkplanes: the data plane is distributed and managed by the DMARs which is closer to the topological of MN, while the control plane, besides the DMARs, reles on a central-mobility entity. The main objective of DMM is exploiting the use of different gateways for traffic with different connectivity and mobility requirements.

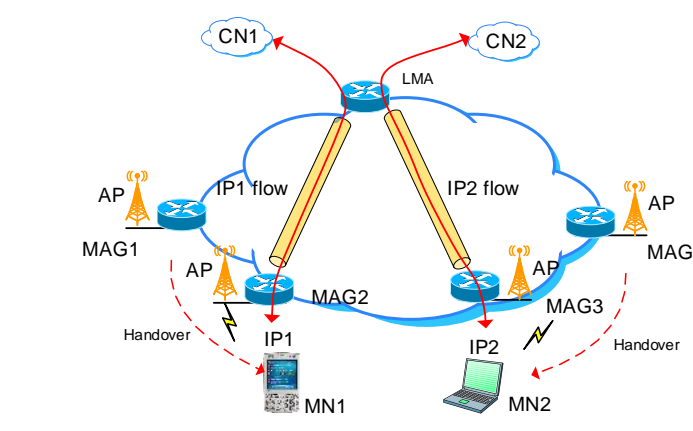

(a) PMIPv6 network architecture

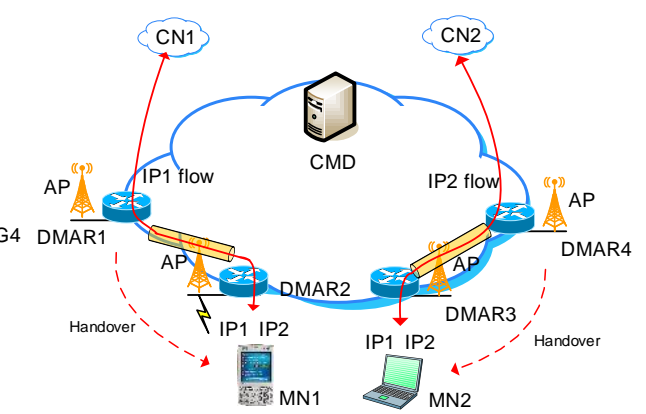

(b) DMM network architecture

Figure 3. Difference Between Pmipv6 and DMM Network Architecture

\subsection{Simulation Environment}

The simulation scenario in OPNET simulator is proposed and illustrated in Figure 4. 


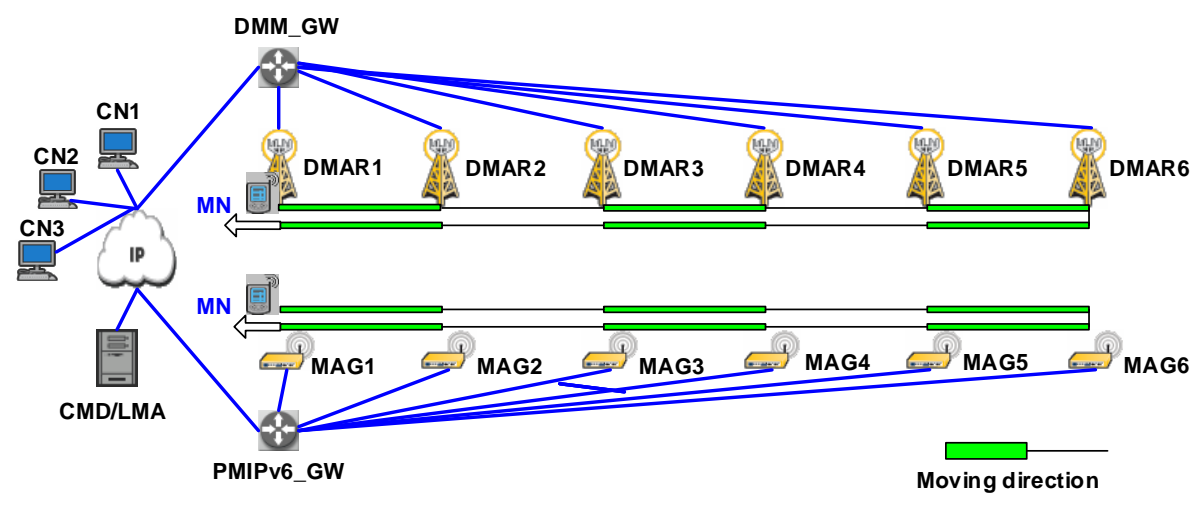

\section{Figure 4. Simulation Architecture Between Pmipv6 and DMM Network}

One of main objectives of DMM is to alleviate the scalability issue of PMIPv6. Another challenge for the DMM solution is related to the frequent movement of MN. An $\mathrm{MN}$ can frequently move to several locations and perform handoffs such that home network prefixed. In order to evaluate the above performances, in Figure 4, one scenario is multiple MNs will move from DMAR1 (MAG1) to DMAR6 (MAG6) in both ways in PMIPv6 network and DMM network. The other seenario is MN will communicate with several CNs while performing multiple handoffs in both networks.

\subsection{Simulation Parameters}

The simulation parameters for two scenarios åne illustrated in Table 1.

\section{Table 1. The Common Simulation Parameters}

\begin{tabular}{|c|c|} 
Paranheters & Value \\
\hline Distance between DMARs and MAGs & $2 \mathrm{~km}$ \\
\hline Traffic Generate Rate (packets/sec) & $0.01,0.025,0.05,0.1, \ldots, 18,20$ \\
\hline Traffic Generate Tine (sec) & $3 \mathrm{~m}, 5 \mathrm{~m}, 7 \mathrm{~m}$ \\
\hline Mobility Speed & $60 \mathrm{~km} / \mathrm{h}$ \\
\hline MN Stants Moving Time & $180 \mathrm{sec}$ \\
\hline Simulation Time & $1380 \mathrm{sec}$
\end{tabular}

\section{Performance Ánalysis and Discussion}

\subsection{Distributed Concept Evaluation}

depicted in Figure 4, MN will move from DMAR1 (MAG1) to DMAR6 (MAG6) and re urn back to the DMAR1 (MAG1). Figure 5 shows the mobility connectivity in PMIPv6/DMM network. It was found that in Figure 5 the continuity of the connectivity was changed. 


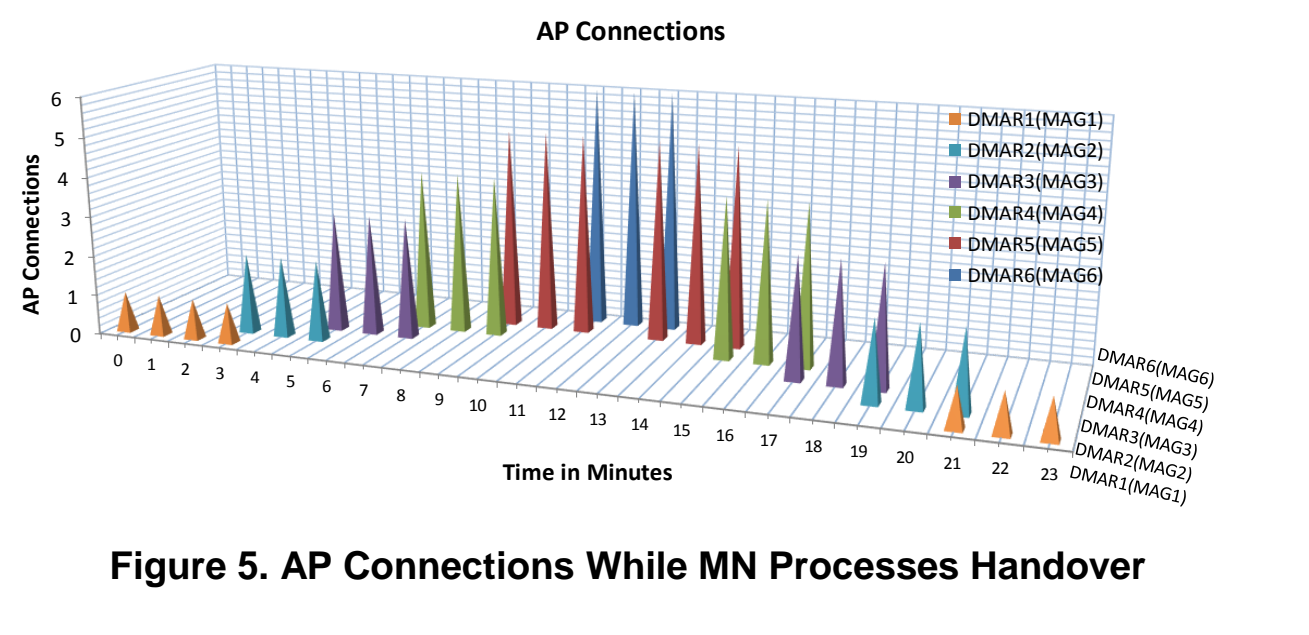

Since the simulation network architecture had the mobility, the Serving ID was changed from number 1 to number 6 as presented in Figure 5, and was then changed back to number 1. It means that MN can support the best comection with DMAR's APs, when MN was moving in PMIPv6/DMM network.

One of the main characteristics of the DMM network is that it will separate data plane and control plane comparing with PMIPv6 metwork. It means the data packet will be distributed on each DMARs, which will not gothrough the In Figure 6, MN sent all tunneled data traffre, which is intercepted by DMAR1 while MN is moving out its home domain (DMAR1). When (AN attached DMAR2-DMAR6 and went back to DMAR2, each new MN's attachment point will receive the tunneled data traffic from DMAR1. It can condlude that the distributed concept is logically correct with a developed DMM node model.

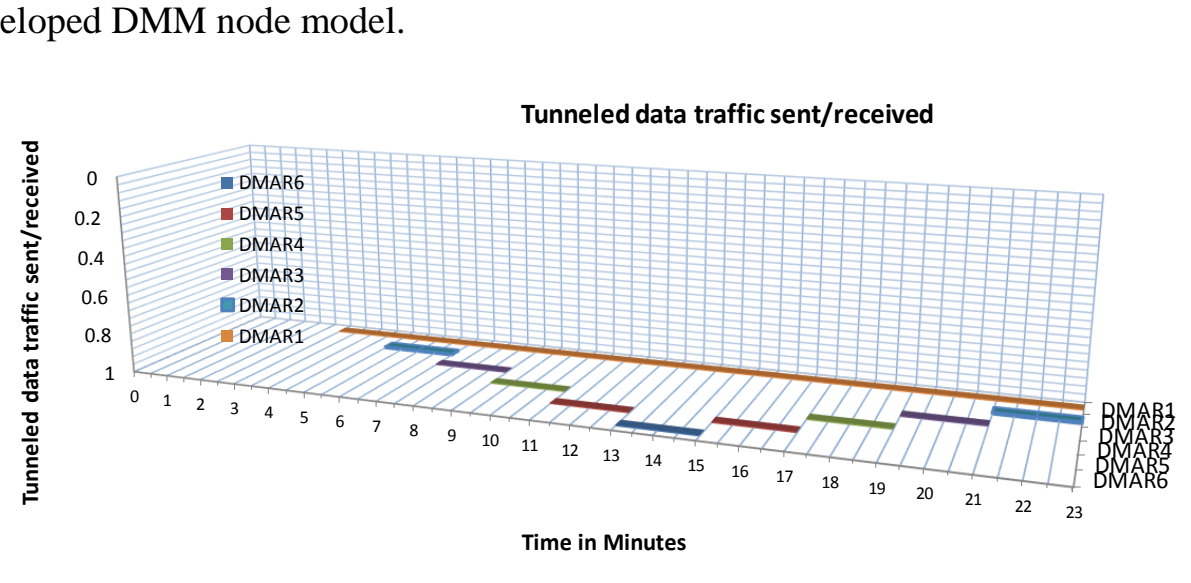

Figure 6. Tunneled Data Traffic Sent/Received in DMM Network

\subsection{Effect of Volumes Data Traffics}

One of the main objectives of DMM is to alleviate the scalability issue of PMIPv6. It means DMM solutions should be able to handle sessions and increasing volumes of mobile data traffic. Therefore, the comparison of CPU utilizations of CMD and LMA and the packet delivery ratio were done and evaluated in DMM and PMIPv6 network, respectively.

The simulation scenario was proposed as $\mathrm{MN}$ communicated with multiple CNs while performing several handoffs. The data traffic generated rate is changed from $0.01 \mathrm{packet} / \mathrm{sec}$ to $20 \mathrm{packets} / \mathrm{sec}$ in order to evaluate the performance of increasing volumes of mobile data traffic. Meanwhile, multiple CNs generate this data traffic at 3 
minutes, 5 minutes and 7 minutes in DMAR1 (MAG1), DMAR2 (MAG2) and DMAR3 (MAG3), respectively. It means MN will communicate with several sessions while $\mathrm{MN}$ in handoffs.

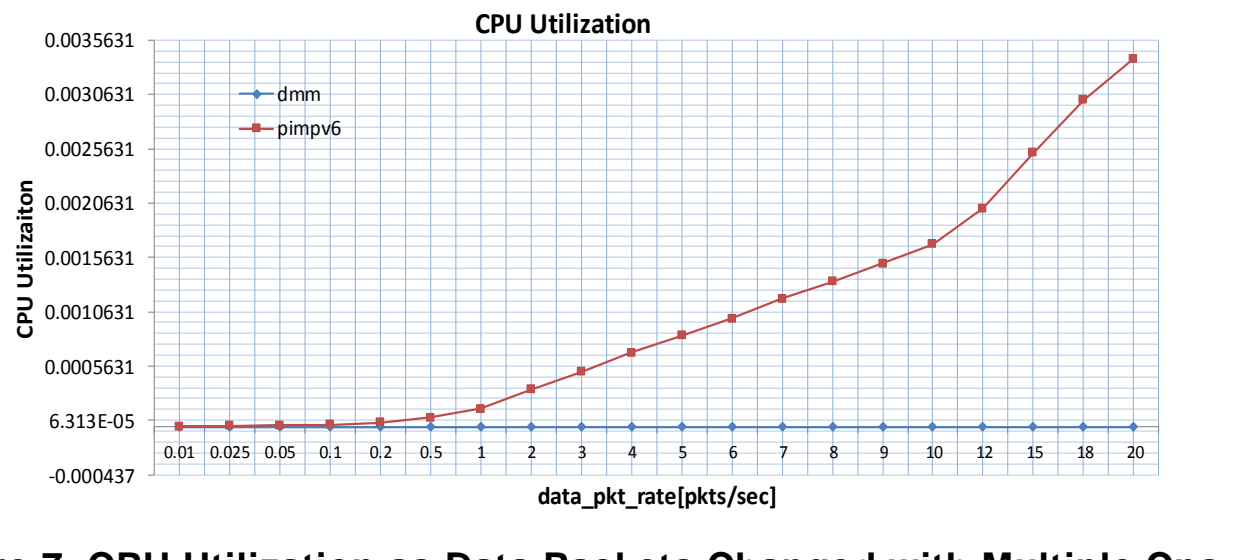

Figure 7. CPU Utilization as Data Packets Changed With Multiple Cns

The simulation results are shown in Figure 7. CPU utilization models the IP packet forwarding delays and application processing delays in the node. In the result graphs, CPU utilization of CMD node in DMM netyork is muchlesser than those of LMA in PMIPv6 because data packets in DMM are distributed across all DMARs, while all data traffic in PMIPv6 are transmitted by way of an LMA It also can be seen that the CPU utilization is not affected by increasing volumes of data traffic.

Packet delivery ratio (PDR) means the ratio between the number of packets delivered to the receiver and the number of packets sem by the source. Figure 8 shows the similar trend of PDR in DMM an PMMIPv6 nework with the increasing volumes of data traffic. It means the PDR is not affected too much as the data traffic varies.

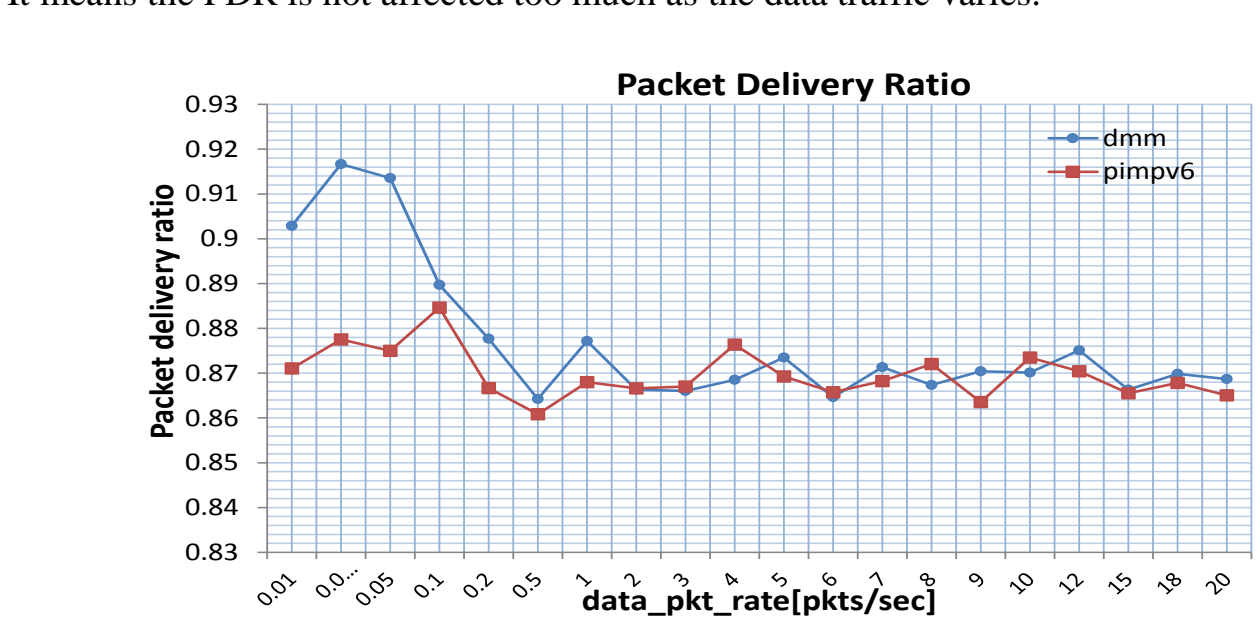

Figure 8. Packet Delivery Ratio as Data Packets Changed with Multiple Cns

\subsection{Effect of Network Size}

The other challenge for DMM solution is related to the frequent movement of MNs and handle with several MNs. It means MNs can frequently move to several locations and performs several handoffs.

The simulation scenario was proposed as the number of MNs vary from 1 to 25 . The data traffic generated rate is run 1packet/sec. In general, the high density of a network size 
may increase the contention and cause network congestion, while also increasing the data traffic lost.

In Figure 9, the CPU utilization in LMA of PMIPv6 network is higher than it is in CMD of DMM network. It also can be seen that CPU utilization will increase sharply as the number of MNs vary from 1 to 25. In DMM network, it seems to not be affected much by varying MNs that seem to increase the volumes of data traffic. The reason is that all data packets will go through the LMA of PMIPv6 between MNs and CNs with the large number of MNs. However, the data packets will not be managed by CMD of DMM according to the properties of DMM solution.

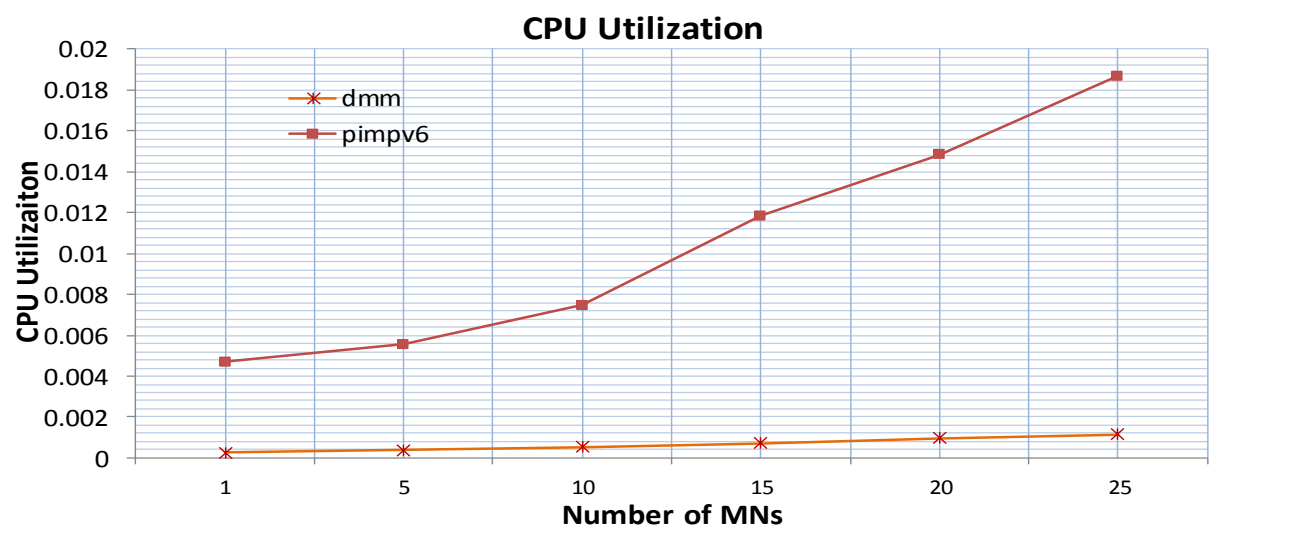

Figure 9. CPU Utilization as Varying Mns from 1 To 25

As depicted in Figure 10, the performance of packet delivery ratio in DMM and PMIPv6 network shows a similar down trend as increasing the volumes of MNs. However, the PDR of DNM performs better than it in PMIPv6 network. With a large number of concurrent/data traffic, extra control traffic causes less available bandwidth for data traffic and increased chances of packet loss due to collisions and interface overflows.

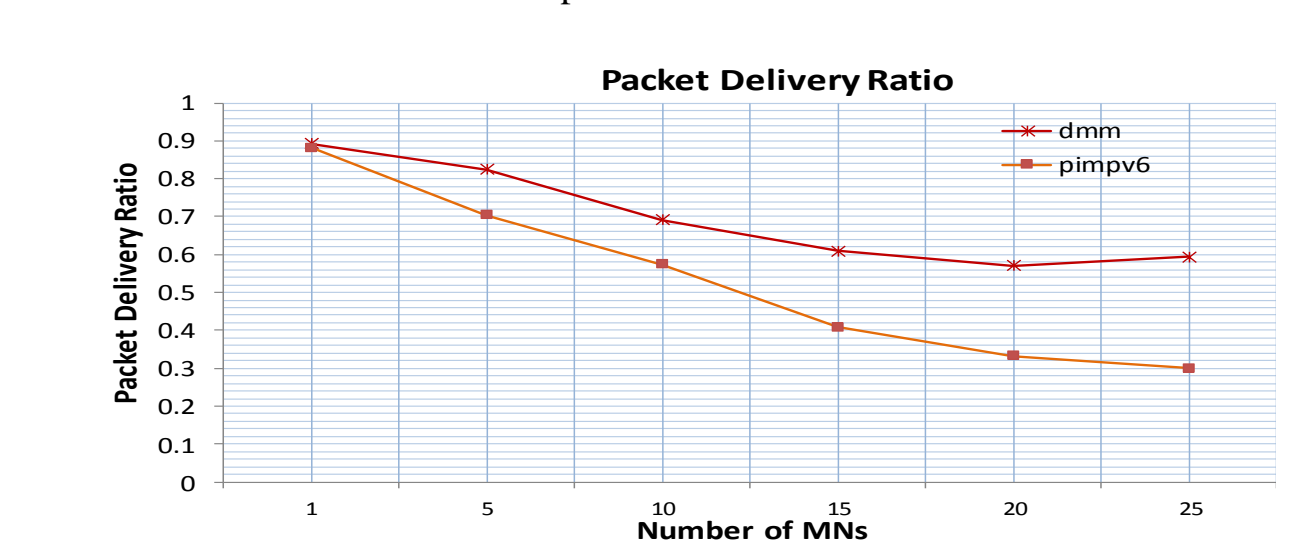

Figure 10. Packet Delivery Ratio as Varying MNs from 1 to 25

\section{Conclusions}

One of the main objectives of DMM is to alleviate the scalability issue of PIMv6. DMM solution should be able to handle several mobile nodes and sessions. Another challenge for DMM solution is related to the frequent movement of mobile nodes. A mobile node can frequently move to several locations and perform several handoffs such that how multiple home network prefixes registered DMAR (both anchor and new 
attached). This leads to a significant impact on how DMARs find the accurate location of mobile node and forward the data packet to the bi-directional tunnel.

This paper shows the main differences and a comparative study between PMIPv6 and DMM network architecture. In order to evaluate the MN's frequent movement and perform several handoffs and several MNs and sessions, the performance of CPU utilization and PDR are implemented in OPNET. As simulation results have shown, while data packets changed with multiple CNs or varying MNs form 1 to 25 , it is concluded that DMM performs good benefits than PMIPv6 in the CPU utilization and PDR.

\section{Acknowledgments}

This research was supported by the Public Welfare Technology Application Project of Science Technology Department of Zhejiang Province (Grant No. 2016031089, 2015C31160), National Natural Science Foundation of China (Grant No. 61373057), Zhejiang Provincial Natural Science Foundation (Grant No. LY14F030095) Humanities and Social Science Research Planning Fund provided by the Ministry of Education of China (Grant No. 13YJA760028), Scientific Research project of Zhejiang Provincial Education (Grant No. Y201534057), Key R \& D projects of science and Technology of Lishui City (Grant No. 20150415, 2015KCPT03), Lishui High-level Personnel Project (Grant No. 2015RC04).

\section{References}

[1] "IMT-2020 5G Vision and Requirement IMTT-2020 (5G) Promotion Group", White Paper, (2014), pp.1-20.

[2] F. Giust, L. Cominardi and C. J.Bernardos, "Distributed Mobility Management for Future 5G Networks: Overview and Analysis of Existing Approaches", IEEE Communication Magazine, vol. 53, no. 1, (2015), pp. 142-149.

[3] H. Chan, D. Liu, P. Seite H. Yokota and Y Korhonen, "Requirements for Distributed Mobility Management", IETF Networking Group RFC 7333, (2014).

[4] D. Liu, Z. Cao, P. Seite and H. Chan, "Distributed mobility management deployment scenario and architecture", IETF DMM Working Group, draft-liu-dmm-deployment-scenario-05 (work in process), (2015).

[5] D. Liu, J. C. Zuniga, P. Seite, H. Chan and C. J. Bernardos, "Distributed Mobility Management: Current practices and gap analysis" $1 E T F$ DMM Working Group, RFC 7429, (2015).

[6] H. Chan, P. Seite, K. Pentikousis and A. Dutta, "Distributed Mobility Management Framework", IETF DMM working Group draft-chan-dmm-framework-03, (2013).

[7] F. Giust, A. de la liva, C. J. Bernardos and R. P. F. D. Costa, "A Network-based Localized Mobility Solution for Distribated Mobility Management", Wireless Personal Multimedia Communications (WPMC), 2011 14th International Symposium on, (2011), pp. 1-5.

[8] C. J. Bennardos, F. Giust and A. de la Oliva, "A PMIPv6-based solution for Distributed Mobility management", IETF DMM Working Group, draft-bernardos-dmm-pmip-06 (work in progress), (2015).

[9] J. Kim, S. Koh, H. Jung and Y. Han, "Use of Proxy Mobile IPv6 for Distributed Mobility Control", IECF $d$ raft-jikim-dmm-pmip-00 (work in progress), (2012).

10] H. Jung, M. Gohar, J. In Kim and S. J. Koh, "Distributed Mobility Control in Proxy Mobile IPv6 Networks", 2011 Institute of Electronics, Information and Communication Engineers (IEICE) TRANS. COMMUN., vol. E94-B, no. 8, (2011), pp. 2216-2224

[11] K. Sun and Y. Kim, "Use case analysis for supporting flow mobility in DMM", IETF Network Working Group, draft-sun-dmm-use-case-analysis-flowmob-dmm-03 (work in process), (2014).

[12] D. H. Shin, D. Moses, M. Venkatachalam and S. Bagchi, "Distributed Mobility Management for Efficient Video Delivery over All-IP Mobile Networks: Competing Approaches”, IEEE network, (2013), pp. 28-33.

[13] F. Gi, C. J. Bernardos and D. L. Oliva, "A. Analytic Evaluation and Experimental Validation of a Network-based IPv6 Distributed Mobility Management Solution”, IEEE Transactions on Mobile Computing, vol. 13, no. 11, (2014), pp. 2484-2497.

[14] J. I. Kim and S. J. Koh, "Distributed Mobility Management in proxy mobile IPv6 using hash function", Information Networking (ICOIN), (2013), pp. 107-112.

[15] Y. Kim, K. Sun and Y. Kim, "Distributed mobility management scheme for the network", The Journal of Korean Institute of Communications and Information Sciences, vol. 11, no. 39C, (2014), pp. 10781087.

[16] OPNET, http://www.opnet.com.tw/, (2016). 
[17] H. A. Ahmad, R. M. Ouzzif and Bertin, "Distributed mobility management: Approaches and Analysis", (2013), pp. 1297-1302.

[18] C. J. Bernardos and J. C. Zuniga, "PMIPv6-based distributed anchoring", IETF Network Working Group, draft-bernardos-dmm-distributed-anchoring-06 (work in process), (2015).

\section{Authors}

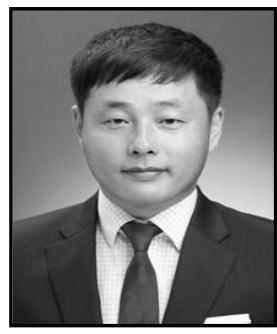

Ye Wang, He received his B.S. in Electrical Engineering and Automation from Beihua University, China in 2003. He joined Zhuhai Wanlida Electric Co., LTD. in 2003 China, and also received his M.S., Ph.D in Information and Communication Engineering from Kongju National University in Korea in 2009, 2013, respectively. Since then, he has been a Lecturer in Lishui University, Zhejiang Province in China. His main research interests include Thternet of Things, Mobile Internet architecture and NGN mobility management.

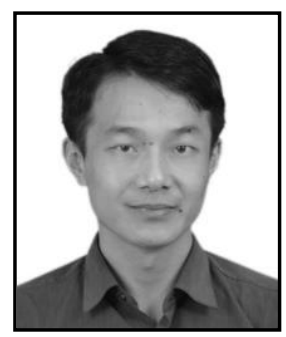

Xiaobo Li, He received his B.SC. in Microelectronics (1990) from Nankai University (China), Master of Engineéring (Research) (2004) from The University of Sydney (Australia) and Ph.D. in Pathology and Pathophysiology (2012) nom Zhejiáng University (China). Now he is a full-time Professor at Department of Computer Science and Technology, College of Engineering, Gishui University, China. His current research interests include different aspects of bioinformatics, machine learning and data mining.

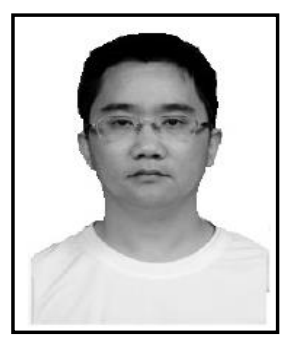

Zhang Wenhui, He was born in 1980 . He received his Ph.D. degree in Harbin Institute of Technology in 2011. Currently, he is a Prelec(or)at Institute of Technology, Lishui University, Lishui City, China.

His research interests include electronic information engineering, cobust control, adaptive and intelligent control and their application.

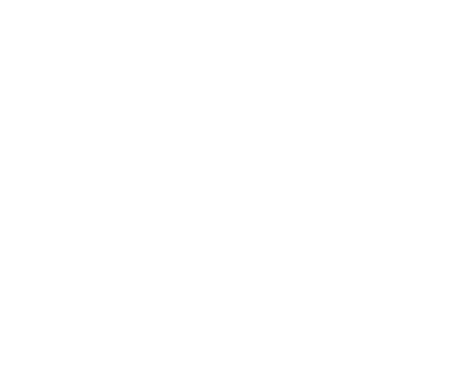


International Journal of Multimedia and Ubiquitous Engineering

Vol.11, No.8 (2016)

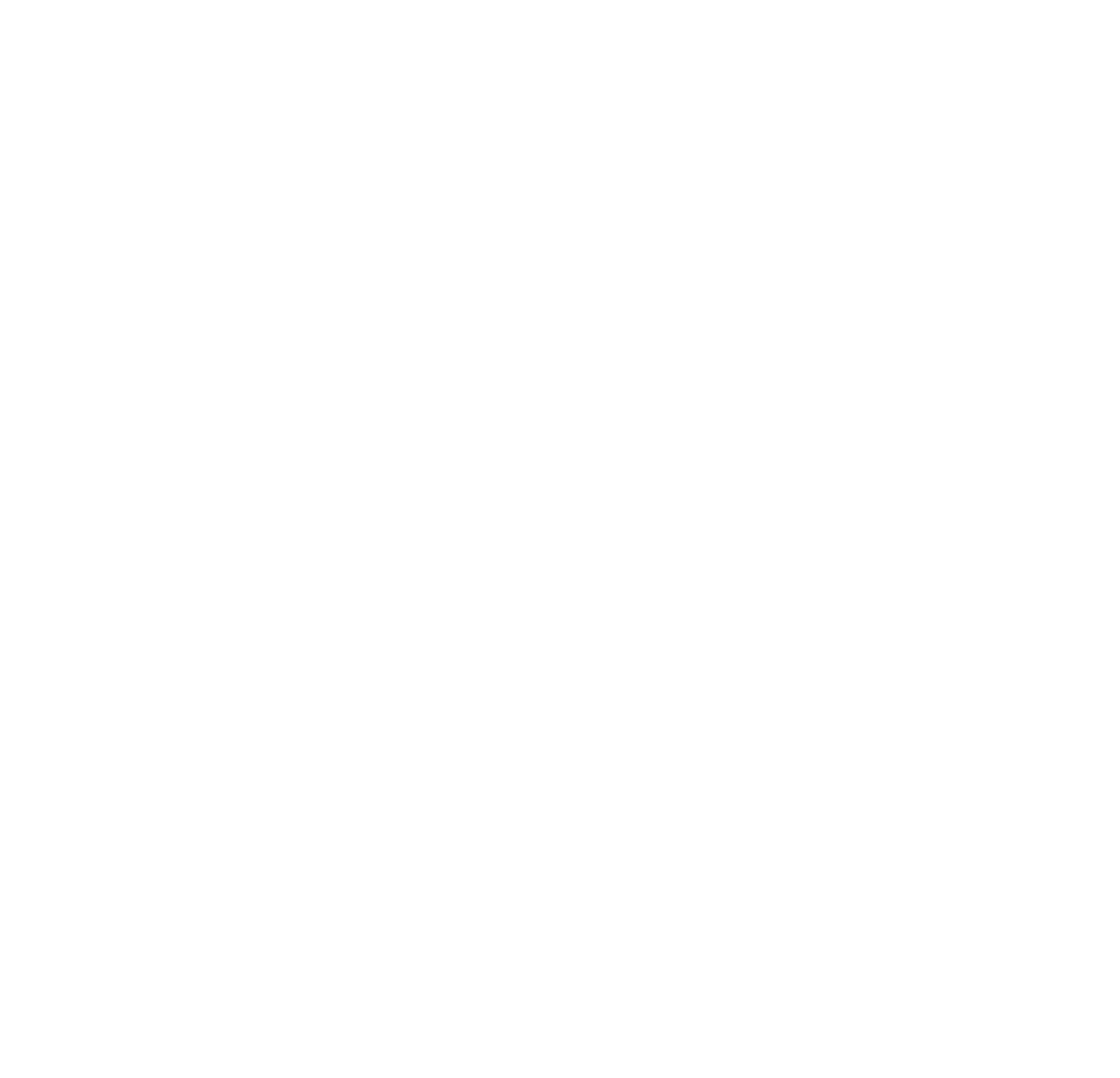

